\title{
Wide Spectrum of Metacarpal Sign: Three Cases of Metacarpal Sign and Its Variants
}

\author{
${ }^{1}$ Saurabh Verma, ${ }^{2}$ Kedar Phadke, ${ }^{3}$ Mukul Mohindra, ${ }^{4}$ Paritosh Gogna, ${ }^{5}$ Jitesh K Jain
}

\begin{abstract}
Metacarpal sign is a diagnostic radiological finding for cases of gonadal dysgenesis, also reported to be associated in a number of other endocrine abnormalities. Technically, it refers to relative difference in the lengths of medial three metacarpals, with the fourth and fifth metacarpals being shorter than the third metacarpal. We incidentally came across three cases that have been mentioned in this report. The first case primarily came to notice because of short fourth and fifth metatarsals. Further evaluation revealed that he also had short fourth and fifth metacarpals. The second case had isolated short fifth metacarpal and was idiopathic. The third patient, a known case of idiopathic hypoparathyroid hormone deficiency, had a short third, fourth, and fifth metacarpal, which is an unreported skeletal phenotype. The latter two cases had slight variation from the classical description of metacarpal sign, and may be a part of still hidden spectrum of metacarpal phenotypes.
\end{abstract}

Keywords: Archibald sign, Metacarpal sign, Short metacarpals.

How to cite this article: Verma S, Phadke K, Mohindra M, Gogna P, Jain JK. Wide Spectrum of Metacarpal Sign: Three Cases of Metacarpal Sign and Its Variants. Int J Recent Surg Med Sci 2017;3(1):53-55.

\section{Source of support: Nil}

Conflict of interest: None

\section{INTRODUCTION}

A positive metacarpal sign means that a tangential line drawn from the distal end of head of fourth and fifth metacarpals passed through the distal end of third metacarpal (Figs 1A and B), while if the same line passes tangential to the head of the third metacarpal, it signifies a borderline metacarpal sign. ${ }^{1-3}$ Normally, the extension of this line passes distal to the head of

\footnotetext{
${ }^{1-3,5}$ Fellow, ${ }^{4}$ Registrar

${ }^{1,2}$ Department of Spine, Indian Spinal Injuries Centre New Delhi, India

${ }^{3,5}$ Department of Arthroscopy, Safdarjung Hospital, New Delhi India

${ }^{4}$ Department of Orthopedics, Pt. Bhagwat Dayal Sharma Post Graduate Institute of Medical Sciences, Rohtak, Haryana India

Corresponding Author: Saurabh Verma, Fellow, Department of Spine, Indian Spinal Injuries Centre, New Delhi, India, Phone: +91-9868409007, e-mail:sau_verma_2001@yahoo.com
}
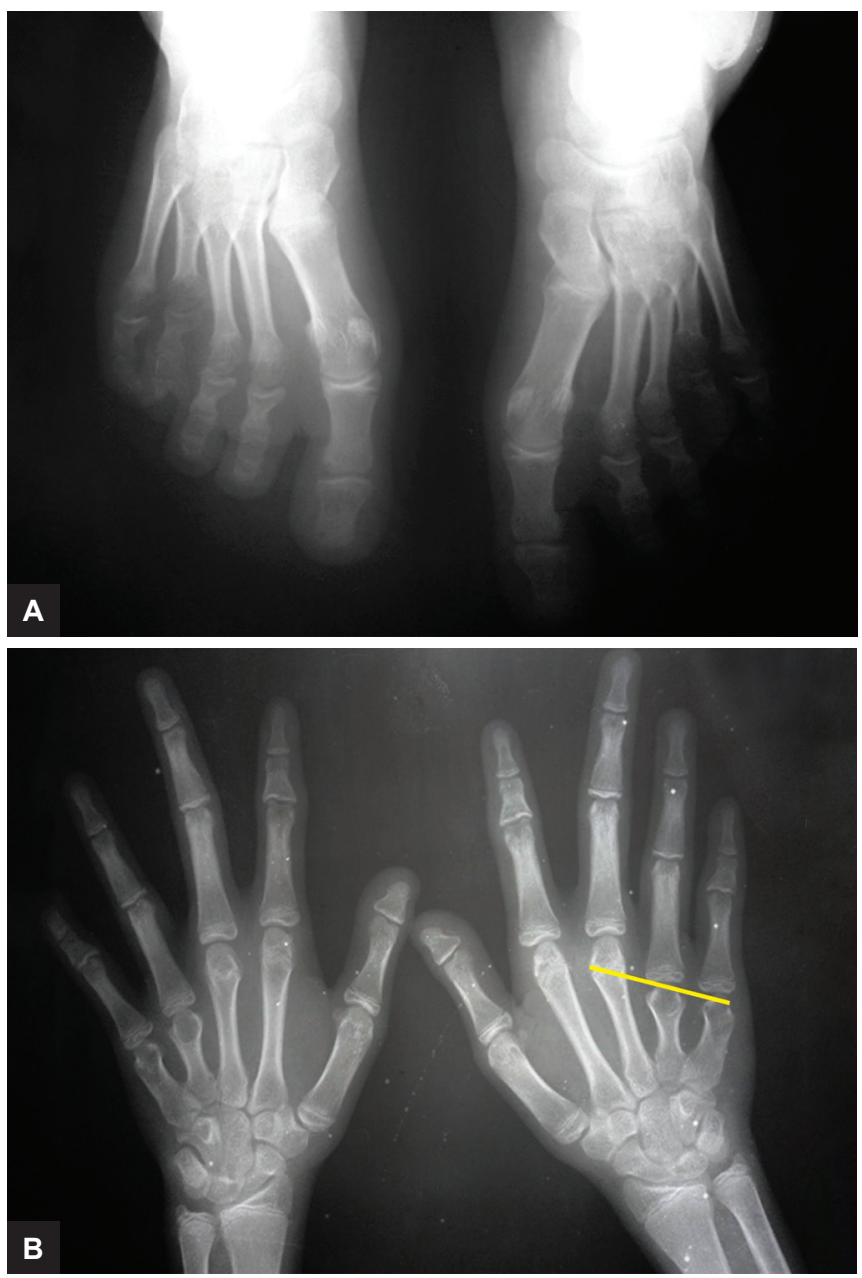

Figs $1 \mathrm{~A}$ and $\mathrm{B}:(\mathrm{A})$ Radiograph of both feet as an anteroposterior view of a 13-year-old female displaying short fourth and fifth metatarsals; and (B) Radiograph of both hands of the same 13-yearold female displaying short fourth and fifth metacarpals (positive metacarpal sign)

the third metacarpal and does not intersect it (negative metacarpal finding). ${ }^{1}$ Various authors have reported this metacarpal finding to be associated with a number of conditions namely Turner Syndrome, Albright's hereditary osteodystrophy, pseudohypoparathyroidism, pseudopseudohypoparathyroidism, basal cell nevus syndrome, sickle cell disease, hereditary multiple exostosis, and homocystinuria. ${ }^{1,2,4-6}$ An incidence of 4 to $10 \%$ of metacarpal sign has been reported in large series. ${ }^{1,2}$ Here, we are reporting three cases of metacarpal sign and its variants. A written informed consent was taken from all the patients for the publication of this case series. 


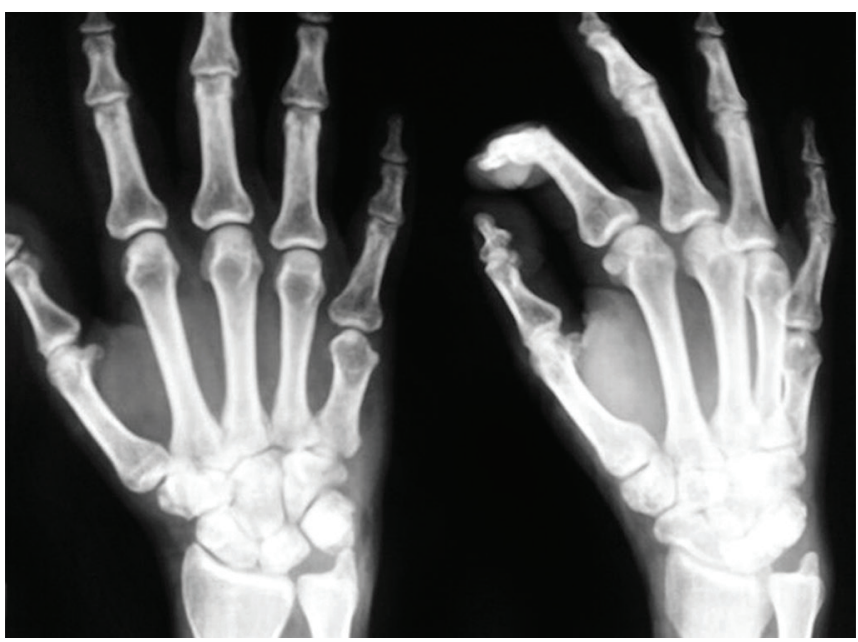

Fig. 2: Radiograph of right hand anteroposterior and oblique views of a 21-year-old male showing isolated short fifth metacarpal

\section{CASE REPORTS}

\section{Case 1}

A 13-year-old female reported to our clinic with history of twisting injury to the left ankle. On getting routine radiographs, bilateral shortening of fourth and fifth metatarsal (Fig. 1A) was found as an incidental finding, with no evidence of any bony injury. Since clinically this patient was observed to have dimpling of skin over medial two knuckles on clenching fist, we went for radiography of hands, and found shortening of fourth and fifth metacarpals (Fig. 1B) as well in both the hands. The patient was thoroughly evaluated for expected associations with the concerned endocrinal abnormalities. There was no history of seizures. The stature seemed normal and the general physical examination was also unremarkable. The serum calcium, serum phosphate, and serum alkaline phosphatase levels were within normal limits, as were serum albumin and 24-hour urinary calcium excretion and thyroid hormone profile. We could neither find any other skeletal abnormality on a skeletal survey nor did the patient have any sort of functional disability.

\section{Case 2}

A 21-year-old male presented with injury to the right index finger. On routine radiography, although no bony injury was seen, an isolated shortened fifth metacarpal was noticed (Fig. 2). The patient was treated conservatively and recovered well. Patient was subjected to the extensive investigation profile mentioned in case 1 , but nothing significant turned out. His skeletal survey was also normal except for the above-mentioned short fifth metacarpal.

\section{Case 3}

A 22-year-old female, with a rounded face, came to the emergency with complaints of pain in the right hand

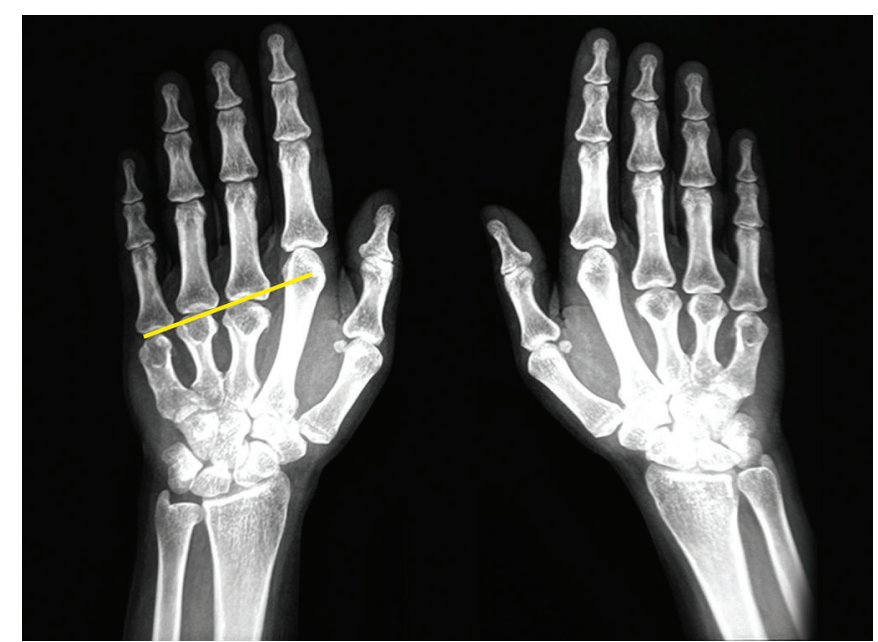

Fig. 3: Radiograph of both hands anteroposterior view of 22-yearold female showing short third, fourth, and fifth metacarpals

following a fall on the ground on outstretched hand. On radiography, there was no bony injury to hand, but finding of a short third metacarpal along with shortened fourth and fifth metacarpals was found in both hands (Fig. 3). A detailed history revealed that patient was a known case of idiopathic parathyroid hormone (IPH) deficiency. The diagnosis had been reached after finding hyperphosphatemia, decreased serum calcium, and parathyroid hormone levels almost a couple of years back.

\section{DISCUSSION}

In their series of examining 2,594 patients, Archibald et $\mathrm{al}^{1}$ found the signs to be positive in $4.2 \%$ of the hands and borderline positive in $4.8 \%$ of hands in patients attending the child growth and endocrine clinics of Rockfeller Hospital. In a series by Bloom, ${ }^{2}$ positive metacarpal finding was seen in $10.7 \%$ of patients out of 1,000 patients they examined.

Valizadeh et $\mathrm{al}^{3}$ suggested that metacarpal shortening is a nonspecific finding in cases of pseudohypoparathyroidism type Ia or pseudopseudohypoparathyroidism and may be found even in cases of idiopathic hypoparathyroidism. This has been found as a normal variant in 2 to $4 \%$ of subjects along with association of short metatarsals in some cases of pseudohypoparathyroidism. ${ }^{2}$ Slater ${ }^{7}$ reported positive metacarpal finding in $9.6 \%$ of an unselected group of 1,510 patients he examined, but could not find any endocrine abnormality in any of them.

The first case we have discussed merits reporting to alert the attending physician regarding the importance of finding incidentally short metatarsals. As per Archibald, this could be a normal variant or could be seen in cases of pseudohypoparathyroidism. ${ }^{1}$ Although in our case, it was found to be idiopathic, this finding warrants radiographs of both hands to search for short metacarpals, and a screening for syndromes associated with positive 
metacarpal finding. At least, clinically, one should look for absence or dimpling of medial two knuckles visible upon asking the patient to clench his fist. ${ }^{2}$ Also, we suggest naming this entity "Archibald Sign" rather than calling it a metacarpal finding. This may avoid expected confusion arising from the varied spectrum of this subtle finding, as its definition and associations also hold true for short fourth and fifth metatarsals and not only metacarpals.

The second rare case presented in this article had an isolated shortened fifth metacarpal. One must, however, note that it is the shortened fourth metacarpal, i.e., the key linkage to the associations listed above. ${ }^{1,2} \mathrm{An}$ isolated short fifth metacarpal has been reported in association with familial variant of type I diabetes mellitus (insulin resistance type I). ${ }^{8}$ Patel and Davies ${ }^{8}$ described three members of an extended family spanning over two generations with insulin resistance Type I and isolated short fifth metacarpals. However, our case could not be ascribed to any association despite meticulous examination and extensive investigations, and was eventually labeled "idiopathic."

The third case we have reported, to the best of our knowledge, is a skeletal phenotype yet undescribed in literature. In this patient, a tangential line drawn from the distal end of head of third, fourth, and fifth metacarpals passed through the distal end of second metacarpal (Fig. 3). Clinically, such cases can be suspected when the middle finger is observed to be shorter than the index finger. The lateral three knuckles were absent upon asking the patient to make a fist. This patient was a known case of IPH deficiency. Earlier, a couple of reports, one by Valizadeh et $\mathrm{al}^{3}$ and another by Isozaki et $\mathrm{al}^{5}$ have predicted an association between IPH deficiency and the classical metacarpal sign. This study implicates that not just fourth and fifth, but any number of short metacarpals warrant a thorough search for the abovementioned associations, but more such cases need to be reported to justify this conclusion. Also, if it so happens, the definition of metacarpal finding, technically, would go incorrect and need to be redefined.

\section{REFERENCES}

1. Archibald RM, Finby N, De Vito F. Endocrine significance of short metacarpals. J Clin Endocrinol Metab 1959 Oct;19:1312-1322.

2. Bloom RA. The metacarpal sign. Br J Radiol 1970 Feb; 43(506):133-135.

3. Valizadeh N, Mehdizadeh A, Nazarbaghi S. Short fourth and fifth metacarpals in a case of idiopathic primary hypoparathyroidism. Indian J Endocrinol Metab 2013 Sep;17(5):924-926.

4. Tzavease A, Paraskevas G, Gekas C, Vrettakos A, Antoniou K, Spyridakis I. Anatomical variation of co-existence of 4 th and 5 th short metacarpal bones, sesamoid ossicles and exostosis of ulna and radius in the same hand: a case report. Cases J 2008 Oct 29;1(1):281.

5. Isozaki O, Sato K, Tsushima T, Shizume K, Takamatsu J. A patient of short stature with idiopathic hypoparathyroidism round face and metacarpal signs. Endocrinol Jpn 1984 Jun;31(3):363-367.

6. Tamburrini O, Bartolomeo-De Iuri A, Andria G, Strisciuglio P, Del Giudice E. Short fourth metacarpal in homocystinuria. Pediatr Radiol 1985;15(3):209-210.

7. Slater S. An evaluation of the metacarpal sign (short fourth metacarpal). Pediatrics 1970;46:468-471.

8. Patel VK, Davies HA. Insulin resistance type A and short 5 th metacarpals. Diabet Med 2003 Jun;20(6):500-504. 